\title{
Reconstruction of Spectra obtained with PYTHEAS through Photometric Methods
}

\author{
M. Gray \\ DASGAL - Observatoire de Paris, 77 avenue Denfert-Rochereau \\ 75014 Paris, France
}

\section{Introduction}

PYTHEAS is an imaging spectrophotometer giving high spectral and spatial resolutions in joining together several optical concepts (Le Coarer et al 1992, and Georgelin et al. in these Proceedings). It will be shown in this paper that, contrary to other spectrographs using somewhat complex methods of data reduction, the PYTHEAS imaging spectrometer requires simple photometric methods in order to reconstruct the spectra of astrophysical objects.

\section{Optical information and photometric methods}

\subsection{Spectral and spatial optical information on the detector.}

The CCD image of a continuous spectral energy distribution for a permanent gap between the layers of the Fabry-Perot consists in a series of channelled spectra : each elementary beam, spectrally filtered by the FP interferometer and sampled by a microlens in the frame, gives light to the whole surface of the grism which separates the various F-P orders by displaying them in a line on the detector. Each line or channelled spectrum consists of a series of Fabry pupils (spectral elements), each of them containing the luminous flux emitted on a certain wavelength by the object under investigation. After scanning the FP interferometer across its free spectral range, we obtain a series of shifted channelled spectra whose set provides us with a chart showing the photometric values of flux according to the wavelength. Consequently, some forms of calibration (continuum lamp, spectral lamp) allow the reconstruction of the spectra of the astrophysical object through simple photometric measures.

\subsection{Reconstruction of spectra through photometry}

PYTHEAS presents spectral and spatial information on the detector without a blemish. Moreover, for optical reasons (telescope, FP, microlens array), the profiles of the Fabry pupils could be assessed through two dimensional Gaussian functions as first approximation. It is essential to know the flux contained in each monochromatic spot with high accuracy to reconstruct the whole spectrum of an elementary area in the field. For this purpose, it is necessary to use a computer program for crowded field stellar photometry, e.g. DAOPHOT (Stetson $1987,1991)$. The power of a computer program as DAOPHOT lies in the profile fitting photometric method exploiting the best characteristics of the two analytic and empirical approaches to determine the Point Spread Function (PSF). 
We describe below the various operations of the DAOPHOT program used for PYTHEAS data reduction in the reconstruction of the spectra of astrophysical objects. This data processing is the same for the images of the field under investigation and for those obtained by calibration. Actually the photometric values obtained by calculating the flux contained in the Fabry pupils are erroneous if we use the PSF proposed by DAOPHOT. This can be explained by the specificity of the images provided by the spectrometer in which the bidimensional profiles of the spots cannot simply be put into the category of Gaussian functions as is the case with the stars. So it would be better to take as bidimensional analytic functions :

- Gauss $\otimes$ Airy, in the direction parallel to the lines of the Fabry pupils.

- Gauss, in the orthogonal direction as regards the Fabry pupils.

50 CCD images of the field under investigation.
50 CCD images obtained by calibration.

(One from each scanning step of the FP interfeometer)

\section{DAOFIND :}

- automatic detection of the positions of the centers in all the Fabry pupils.

- use of truncated lowered Gaussian functions with criteria giving greater importance to the properties of the centers than those of the wings.

\section{PHOT :}

- measurement of the magnitude of each Fabry pupil with simple aperture photometry. The assessment of the sky brightness (area, value) is crucial.

\section{PSF :}

- determination of the two-dimensional Point Spread Function in selecting the brightest, least crowded "stars" (Fabry Pupils). This operation is reiterated so as to improve the Signal to Noise Ratio (SNR).

- With this model of PSF determined for each CCD image, and thanks to various procedures using profile fitting photometry, the photometric values of the flux contained in each Fabry Pupil can considerably be improved.

\section{Conclusion}

The PYTHEAS imaging spectro-photometer enables us to have a spatial sampling of the light beam by disconnecting spectral information from spatial information. Thus, for spectra reconstruction, we can use simple photometric methods similar to the methods used in crowded field stellar photometry. A computer program as DAOPHOT could be well-adapted to this photometric work in PYTHEAS data reduction, thanks to a well- adapted two dimensional profile fitting for the PSF. 


\section{References}

E. Le Coarer, Ph D Thesis, University of Paris VII, 1992.

E. Le Coarer, Y.P. Georgelin, G. Monnet, CRASP, C 315 serie II p 45, 1992.

E. Le Coarer, et al., ESO High Resolution Spectroscopy Working Group, 1992.

E. Le Coarer, et al., ESO Worshop on High Resolution Spectroscopy with the VLT, 1992.

E. Le Coarer, et al., A. \& A., 1994 (submitted).

P.B. Stetson, Pub. Astr. Soc. Pac., 99, 191-222, 1987.

P.B. Stetson, 3rd ESO/ST-ECF Data Analysis Workshop 187-199, April 1991. 